\title{
Phosphorescent materials for application to organic light emitting devices*
}

\author{
M. A. Baldo ${ }^{1}$, M. E. Thompson ${ }^{2}$ and S. R. Forrest ${ }^{1, \dagger}$ \\ ${ }^{1}$ Center for Photonics and Optoelectronic Materials (POEM), Department of Electrical \\ Engineering and the Princeton Materials Institute, Princeton University, Princeton, NJ \\ 08544, USA \\ ${ }^{2}$ Department of Chemistry, University of Southern California, Los Angeles, California \\ 90089, USA
}

\begin{abstract}
Organic phosphors have demanded the attention of the organic electroluminescence community because they enable efficiencies quadruple that of fluorescent materials. In this work, we review the categories of organic phosphors: lanthanide complexes, organic phosphors and metal-organic complexes. The characteristics necessary for efficient phosphorescence are considered and conclusions are drawn as to the most promising materials.
\end{abstract}

\section{INTRODUCTION}

The application of phosphors to light emitting devices has a well-established precedent: the cathode ray tube, where luminescence is obtained from atomic transitions within carefully selected inorganic materials. Similar techniques can be used to exploit the advantages of phosphorescence in the flourishing field of organic electroluminescence, but the flexibility of organic materials allows for a variety of additional approaches. We shall examine these approaches in this review.

Phosphorescence is distinguished from fluorescence by the speed of the electronic transition that generates luminescence. Both processes require the relaxation of an excited state to the ground state, but in phosphorescence the transition is 'forbidden' and as a consequence it is slower than fluorescence, which arises from allowed transitions. Indeed, phosphorescence may persist for several seconds after a phosphorescent material is excited, whereas fluorescent lifetimes are typically on the order of nanoseconds.

Interest in phosphorescence, and the phosphorescence of organic materials in particular, arises from the application of these materials to organic light emitting devices [1-5] (OLEDs), where it is found that the luminous efficiency may be improved by up to a factor of four over that obtained using fluorescence. This increase is fundamental to organic materials and arises during the formation of an excited state (or exciton) from the combination of electrons and holes. Typically these excitons are localized on a small molecule, or localized to a region of a polymer chain, and hence it is often convenient to describe excitons as particles. For example, an exciton possesses spin, which must be conserved during the emission of a photon. As the ground state is generally spin antisymmetric with a total spin of $S=0$, the decay of $S=0$ excitons is allowed. In contrast, the decay of $S=1$ excitons is not allowed. This poses an obstacle to efficient luminescence because the combination of an electron and a hole with uncorrelated spins is three times as likely to result in a spin-symmetric as opposed to a spin antisymmetric state [6]. From the multiplicity of the exciton spin states, $\mathrm{S}=1$ excitons are known as triplets, and $\mathrm{S}=0$ excitons are singlets. Thus, if the energy contained in the triplet excitons cannot be directed to luminescence, the efficiency of an OLED is reduced by $75 \%$.

Fortunately, although the decay of a triplet state is disallowed by the conservation of spin symmetry, it is occasionally observed if the triplet state is perturbed such that the transition becomes weakly allowed.

\footnotetext{
*Lecture presented at the 4th International Symposium on Functional Dyes-Science and Technology of Functional $\pi$-Electron Systems, Osaka, Japan, 31 May-4 June 1999, pp. 2009-2160.

${ }^{\dagger}$ Corresponding author: E-mail: forrest@EE.Princeton.EDU
} 
In this case, the decay of the triplet state may still be very slow, but phosphorescence is generated. But efficient phosphorescence is rare at room temperature, and attempting to find a material that also readily transports charge is a challenging task. Moreover, very few materials luminesce efficiently in homogenous films due to the quenching of emission by surrounding molecules. The solution to these demands on OLED materials is found by doping the luminescent material into a charge transport host material [7]. Emission then occurs in one of two ways: either by direct carrier trapping and exciton formation on the luminescent dye, or by exciton formation in the host and energy transfer to the luminescent guest. Hence, it is not sufficient merely for the guest to be phosphorescent from its triplet states; it must also be able to gather the triplets formed by electrical excitation.

\section{ENERGY TRANSFER}

There are two mechanisms for the transfer of energy in these materials: Förster and Dexter transfer [8]. Förster transfer [9] is a long range $(\approx 40 \AA-100 \AA)$, non-radiative, dipole-dipole coupling of donor (D) and acceptor (A) molecules. Since it requires that the transitions from the ground to the excited states be allowed for both $\mathrm{D}$ and A species, this mechanism only transfers energy to the singlet state of the acceptor molecule via:

$$
\begin{gathered}
{ }^{1} D^{*}+{ }^{1} A \rightarrow{ }^{1} D+{ }^{1} A^{*} \\
\text { or, } \\
{ }^{3} D^{*}+{ }^{1} A \rightarrow{ }^{1} D+{ }^{1} A^{*}
\end{gathered}
$$

Frequently, phosphorescent dyes possess strong intersystem crossing (ISC) from the singlet to the triplet excited state. For these materials, little or no fluorescence is observed from the singlet state of the acceptor $\left({ }^{1} A^{*}\right)$, and all excited states in the donor are ultimately transferred to the triplet acceptor state $\left({ }^{3} A^{*}\right)$. Typically the donor exciton must also be a singlet to participate, however, there is an exception for donor materials where a triplet-to-ground state transition is weakly allowed (see Eqn 2) [9,10]. For example, energy transfer from a triplet to a singlet state has been demonstrated when the donor is an efficient phosphor [11]. Here, the slower rate of energy transfer from a weakly allowed transition is compensated by the long lifetime of the donor exciton.

Dexter transfer [12] is a short-range process where excitons diffuse from D to A sites via intermolecular electron exchange. In contrast to Förster transfer, Dexter processes require only that the total spin of the D-A pair be conserved under the Wigner-Witmer selection rules [8], via:

$$
\begin{gathered}
{ }^{1} D^{*}+{ }^{1} A \rightarrow{ }^{1} D+{ }^{1} A^{*} \\
\text { or, } \\
{ }^{3} D^{*}+{ }^{1} A \rightarrow{ }^{1} D+{ }^{3} A^{*}
\end{gathered}
$$

Thus, Dexter transfer permits both singlet-singlet and triplet-triplet transfers. However, Förster transfer dominates singlet-singlet transfer at low acceptor concentrations because it is faster over long distances. Energy transfer to phosphorescent dyes then proceeds by Förster transfer of singlets and Dexter transfer of triplets. Dexter transfer processes are slow for all interactions except those between neighboring molecules; thus it is possible that direct charge trapping and exciton formation on a phosphorescent dye may be an equally effective method for generating efficient phosphorescence.

\section{Lanthanide complexes}

The previous discussion of excitons applies to organic materials, but as discussed in the introduction phosphorescence is also possible in 'disallowed' atomic transitions. The most efficient examples of phosphorescence from an atomic species are the lanthanide complexes [13]. Since these transitions possess very sharp spectral bands, near monochromatic or 'saturated' luminescence results. This is obviously also desirable in OLEDs, and examples are red-emitting complexes $[14,15]$ of $^{E^{3+}}$, greenemitting complexes [5] of $\mathrm{Tb}^{3+}$, and more recently $1.54 \mu \mathrm{m}$ electroluminescence has been generated [16] from $\mathrm{Er}^{3+}$, although the efficiencies in all cases have been low (typically $<1 \%$ ). It is illustrative to examine the most successful application [15] yet of a lanthanide complex to OLEDs. Tris(1,3-diphenyl1,3-propanediono)(monophenanthroline) $\mathrm{Eu}(\mathrm{III})\left(\mathrm{Eu}(\mathrm{DBM})_{3}(\mathrm{Phen})\right)$ exhibits red-orange phosphorescence 
at $614 \mathrm{~nm}$ and has been used to generate electroluminesce in organic devices. The emission mechanism is the 'forbidden' ${ }^{5} \mathrm{D}_{0} \rightarrow{ }^{7} \mathrm{~F}_{2}$ transition of $\mathrm{Eu}^{3+}$ which has a radiative lifetime $\approx 100 \mu \mathrm{s}$. In OLEDs, $\mathrm{Eu}(\mathrm{DBM})_{3}(\mathrm{Phen})$ is preferably codeposited with a charge transport material such as [17] biphenyl- $p-(t-$ butyl)phenyl-1,3,4-oxadiazole (PBD), thereby reducing self-quenching and improving charge carrier mobilities.

Approximate energy levels of the $\mathrm{Eu}(\mathrm{DBM})_{3}(\mathrm{Phen})$ :PBD system are shown [18] in Fig. 1. Excitation of the $\mathrm{Eu}^{3+}$ ion occurs via the triplet energy level of the $\mathrm{Eu}^{3+}$ complex ligand [18]. As discussed in Section I, for high efficiency, it is necessary that both host singlets and triplets be transferred to the $\mathrm{Eu}^{3+}$ ion. Förster transfer of singlets is possible [15], as demonstrated by the observation that photoexcitation of PBD results in $\mathrm{Eu}^{3+}$ phosphorescence. However, since the relevant triplet energy levels in the host and the guest triplet state are frequently unknown and, moreover, are difficult to quantify due to their small oscillator strengths, optimizing guest-host systems for resonant triplet transfer is problematic. In the case of $\mathrm{Eu}(\mathrm{DBM})_{3}(\mathrm{Phen})$, the energy of ligand phosphorescence [18] is large $(\approx 2.6 \mathrm{eV})$ and the triplet absorption energy is expected to be even higher. Hence triplet transfer from the host to the ligand requires a host triplet of sufficiently high energy to be in resonance with the guest, and a device structure that minimizes nonradiative losses of host triplets. Analysis of $\mathrm{Eu}(\mathrm{DBM})_{3}(\mathrm{Phen}): \mathrm{PBD}$ is difficult because the triplet energy in PBD is unknown. However, the maximum quantum efficiency [15] of $\mathrm{Eu}(\mathrm{DBM})_{3}(\mathrm{Phen}): \mathrm{PBD}$ is $\approx 1 \%$. Thus, it is suspected that in this material system, energy transfer and particularly triplet-triplet transfer is poor.
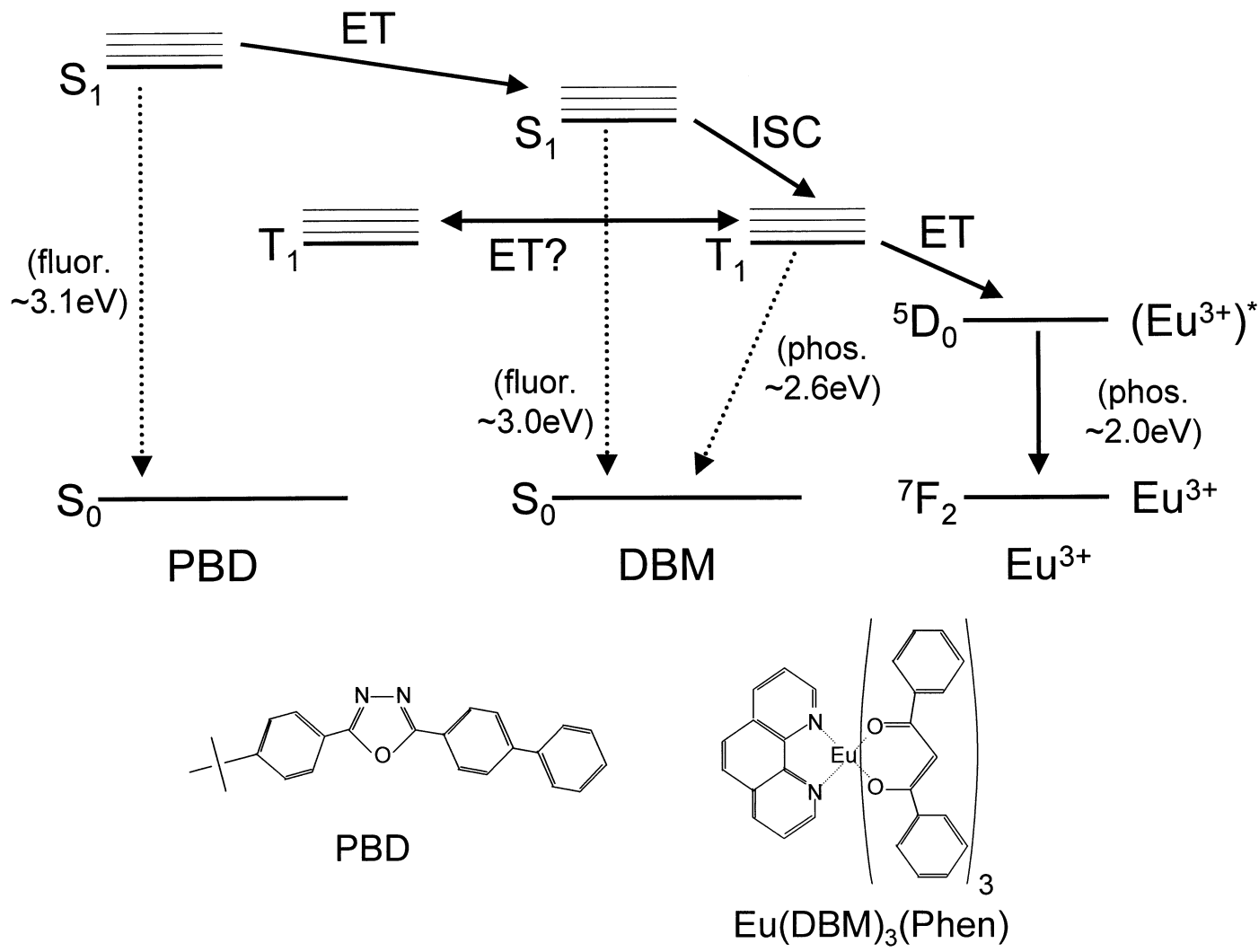

Fig. 1 The chemical structures of the lanthanide complex $\operatorname{Eu}(\mathrm{DBM})_{3}(\mathrm{Phen})$ and a host material PBD. Also shown is a proposed energy level diagram (adapted from [13]) indicating the energy transfer process. Note that the triplet energy level of PBD is unknown, complicating analysis of this system.

Despite the fact that lanthanide materials should be relatively insensitive to triplet-triplet annihilation, quantum efficiency is nevertheless found to decrease as current density increases, and consequently the maximum luminance [15] of the $\mathrm{Eu}^{3+}$ complex is only $460 \mathrm{~cd} / \mathrm{m}^{2}$. Notwithstanding these deficiencies, the saturated emission of lanthanide-based complexes remains extremely attractive for many luminescent 
applications. In the case of vacuum deposited OLEDs, molecular design is required to ensure that the complexes can withstand vacuum sublimation. It is also necessary to minimize the distance between the ligand and the central lanthanide atom to maximize energy transfer within the complex [19]. Then if a host material is chosen for effective energy transfer to a lanthanide complex, or efficient charge trapping on the dye, the uniquely saturated emission of lanthanide complexes will be coupled with high electroluminescent efficiencies.

\section{Purely organic phosphors}

Another example of a phosphorescent material applied to OLEDs is benzophenone [4]. This material has phosphorescent emission at $\lambda \approx 450 \mathrm{~nm}$ and is frequently employed in the study of organic triplet excitons [8]. Benzophenone emits from a triplet state; thus the additional process in the lanthanide complexes of energy transfer from the ligand triplet to the ionic excited state is not required. In general, removing a step from the energy transfer process reduces losses and lowers the inital energy required for excitons to propagate from the host to emissive state of the dye.

Phosphorescence in benzophenone arises from a forbidden transition from excited $\left(\pi^{*}\right)$ to ground $(n)$ states. Spin-orbit coupling is enhanced [8] since both states are localized on the carbonyl group, hence the $\pi^{*} \leftarrow n$ transition becomes weakly allowed. However, the rate of phosphorescence is slow and must compete with non-radiative transitions that acquire intensity by coupling to the vibrations of the molecule [20]. Consequently, phosphorescence from benzophenone is strongly temperature dependent. When employed in OLEDs (see Fig. 2), benzophenone was spun cast in poly(methylmethacrylate) (PMMA). It exhibited negligible phosphorescence at room temperature, however a $\approx 900$-fold increase in quantum efficiency was observed [4] by reducing the temperature to $100 \mathrm{~K}$. A lifetime of $\approx 5.3 \mathrm{~ms}$ at $100 \mathrm{~K}$ has been observed for $1.7 \%$ benzophenone doped into PMMA. Evidently, at room temperature the rate of phosphorescence in benzophenone is too slow to compete with thermally activated non-radiative modes.

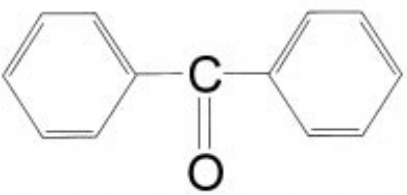

Benzophenone

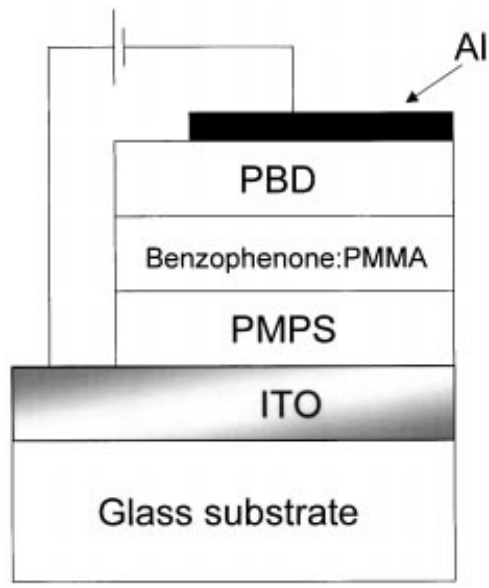

Fig. 2 The chemical structure of the triplet emitter benzophenone and the device structure of the OLED used to obtain phosphorescence at $77 \mathrm{~K}$. Poly(methylmethacrylate) (PMMA) serves as the host for benzophenone; the hole transporting layer is poly(methylphenylsilane) (PMPS) and the electron transport and hole blocking layer is PBD. From [4].

\section{Organometallic phosphors}

The example of benzophenone demonstrates that for efficient room temperature phosphorescence from organic ligands, short triplet lifetimes are required. This may be achieved by spin-orbit (L-S) coupling which mixes singlet and triplet excited states [20]. Spin-orbit coupling is significantly enhanced by the presence of a heavy atom in an organometallic complex. In contrast to the lanthanide complexes discussed earlier, these materials, which typically are complexes of $\mathrm{Os}, \mathrm{Ru}, \mathrm{Pd}, \mathrm{Pt}$, Ir or $\mathrm{Au}$, do not luminesce from an atomic transition. Rather, the lowest energy excited state is frequently a metal-ligand charge-transfer triplet state, mixed with the excited singlet state by L-S coupling. Consequently, 
phosphorescent lifetimes are short $(<100 \mu \mathrm{s})$ and high photoluminescent efficiencies are possible. The mixing between singlet and triplet excited states is also responsible for very high probabilities (>99\%) of intersystem crossing. Thus, both singlet and triplet excitations of these complexes can result in phosphorescent emission.

Because these materials emit from a triplet state, and because the ground state of molecular oxygen is also a triplet, oxygen reversibly quenches phosphorescence by triplet-triplet annihilation. Some complexes may also be quenched by electron transfer and other processes, hence many suitable organometallic complexes have been studied for use as oxygen sensors [21,22]. Application of organometallic complexes in OLEDs was demonstrated [2] by Ma et al., doping several osmium complexes in poly ( $N$-vinyl carbazole). Although very low electroluminescent quantum efficiencies $(<0.1 \%)$ were reported, it was shown that this promising class of materials can ultimately yield efficiency improvements in OLEDs. Recently [23] these workers also demonstrated two new organometallic phosphors based on either gold(I) or copper(I). Both phosphors exhibited strong intersystem crossing and high photoluminescent efficiencies of $23 \%$ and $42 \%$, respectively. But once again, owing to poor energy collection by the phosphor, only low quantum efficiencies $(<0.1 \%)$ were obtained.

High efficiency electroluminescence from an organometallic complex triplet state was ultimately demonstrated [1] in OLEDs using the phosphorescent dye 2,3,7,8,12,13,17,18-octaethyl-21H,23Hporphine platinum (II) (PtOEP). Porphyrin complexes are known to possess long-lived triplet states useful in oxygen detection [24]. The addition of platinum to the porphine ring reduces the phosphorescence lifetime by increasing L-S coupling; the triplet states gain additional singlet character and vice versa. This also enhances the efficiency of intersystem crossing from the first singlet excited state to the triplet excited state. Transient absorption spectroscopy gives a singlet lifetime in PtOEP of $\approx 1 \mathrm{ps}$, and the fluorescence efficiency is extremely weak [25]. In contrast, the room temperature phosphorescence efficiency of PtOEP in a polystyrene matrix is [21] 50\% with an observed lifetime of $91 \mu \mathrm{s}$. Thus, both singlet and triplet excitations in PtOEP yield efficient phosphorescence. Consequently, no significant emission is found for the previously identified singlet state, expected at approximately $580 \mathrm{~nm}$ [25], but as shown in Fig. 3, strong emission is observed from the triplet excited state of $650 \mathrm{~nm}$, with weaker emission at the vibronic harmonic overtones at $623 \mathrm{~nm}, 687 \mathrm{~nm}$ and $720 \mathrm{~nm}$. The emission of PtOEP at $650 \mathrm{~nm}$ is almost as sharp and saturated as the atomic transition of $\mathrm{Eu}^{3+}$ at $614 \mathrm{~nm}$. However, PtOEP is a much deeper red and possesses Commission Internationale de L'Éclairage (CIE) chromaticity coordinates of $(x, y)=(0.72,0.29)$.

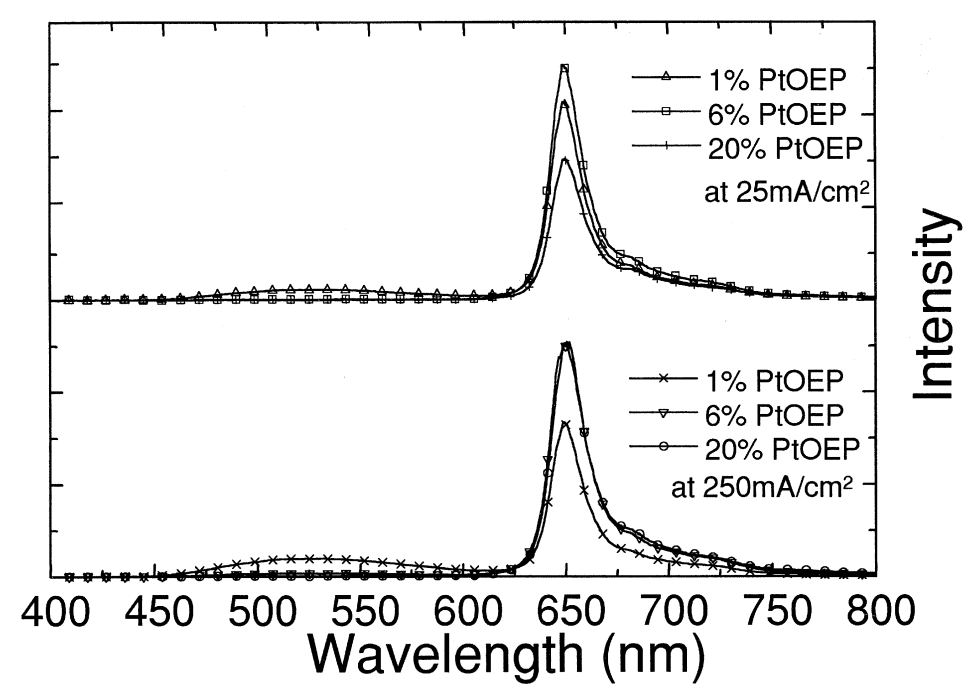

Fig. 3 Spectra of phosphorescent OLEDs with different molar concentrations of PtOEP in $\mathrm{Alq}_{3}$ at different current densities. (a) 1\%,6\% and 20\% PtOEP in Alq ${ }_{3}$ OLEDs at $25 \mathrm{~mA} / \mathrm{cm}^{2}$ (b) $1 \%, 6 \%$ and $20 \% \mathrm{PtOEP}_{\text {in }} \mathrm{Alq}_{3}$ OLEDs at $250 \mathrm{~mA} / \mathrm{cm}^{2}$. Note the increased $\mathrm{Alq}_{3}$ emission at $530 \mathrm{~nm}$ in the $1 \%$ PtOEP OLED due to saturation of PtOEP sites and poor energy transfer. From [1]. 
Conclusive evidence for triplet transfer to PtOEP from the well-known electron transport material tris(8-hydroxyquinoline) aluminum $\left(\mathrm{Alq}_{3}\right)$ was obtained by examining the unnormalized spectra of the two devices in Fig. 4. A $100 \AA$ thick layer of $\mathrm{Alq}_{3}$ doped with $\approx 1 \%$ of the fluorescent dye DCM2 is placed at the heterojunction between $\mathrm{Alq}_{3}$ and 4,4'-bis[N-(1-napthyl)-N-phenyl-amino] biphenyl ( $\alpha$ NPD). $\mathrm{Alq}_{3}$ preferentially transports electrons, whereas $\alpha$-NPD preferentially transports holes; thus exciton formation is localized to this interface. Since DCM2 exhibits efficient energy transfer from $\mathrm{Alq}_{3}$ [26], the $1 \%$ DCM2 in $\mathrm{Alq}_{3}$ layer effectively removes singlet excitons. Remaining singlets eventually recombine in $\mathrm{Alq}_{3}$, yielding the small shoulder in the spectra at $\approx 530 \mathrm{~nm}$. However, in device 2 , an additional layer of $\approx 10 \% \mathrm{PtOEP}$ in $\mathrm{Alq}_{3}$ is introduced $200 \AA$ away from the exciton formation zone. In this device, emission is seen from PtOEP without any change in the intensity of emission from either DCM2 or $\mathrm{Alq}_{3}$. Hence, PtOEP cannot be an efficient electron trap, since carriers removed by PtOEP in device 2 would result in a decrease in the DCM2 and $\mathrm{Alq}_{3}$ emission: an effect clearly not observed. Since the DCM2 acts as a 'filter' that removes singlet Alq 3 excitons, the only possible origin of the PtOEP luminescence is $\mathrm{Alq}_{3}$ triplet states that have diffused through the DCM2 and intervening $\mathrm{Alq}_{3}$ layers.

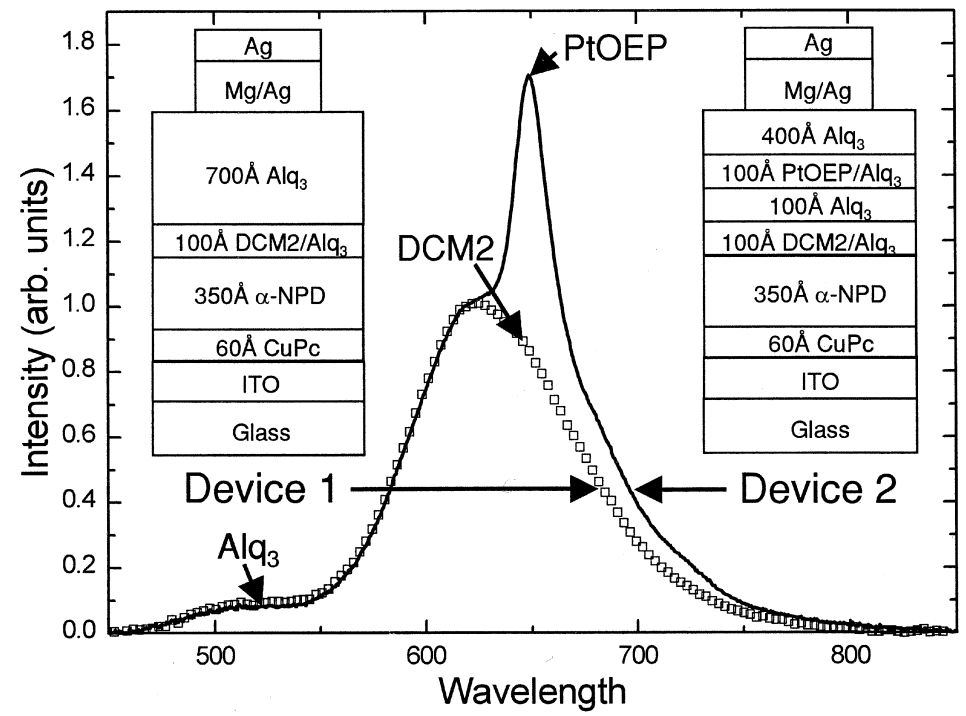

Fig. 4 Two electroluminescent devices demonstrating that $\mathrm{Alq}_{3}$ triplets are transferred to PtOEP. Each device contains a $100 \AA$ thick layer of $\approx 1 \% \mathrm{DCM} 2$ in $\mathrm{Alq}_{3}$ at the recombination zone. This layer acts to remove singlet states. Remaining singlets recombine in $\mathrm{Alq}_{3}$, yielding the shoulder apparent in the spectra at $530 \mathrm{~nm}$. Device 2 contains an additional layer of $\approx 10 \%$ PtOEP in $\mathrm{Alq}_{3}$ positioned $200 \AA$ away from the $\mathrm{Alq}_{3} / \alpha-\mathrm{NPD}$ interface. Strong emission is seen from the PtOEP without a corresponding decrease in emission from DCM2 or Alq 3 . The spectra were measured at a current density of $6 \mathrm{~mA} / \mathrm{cm}^{2}$. From [1].

An alternative demonstration of triplet transfer to PtOEP was described by Cleave et al. [3]. Here, PtOEP was doped into the polymer host poly[4-(N-4-vinylbenzyloxyethyl,N-methylamino)-N-(2,5-ditert-butylphenylnapthalimide)] (PNP) and the transient electroluminescence was examined in OLEDs with the general structure ITO/polyvinylcarbazole/PtOEP:PNP/calcium [3]. The observed decay rate is the convolution of the rate of energy transfer from PNP to PtOEP and the PtOEP triplet relaxation rate. Analysis of the electroluminescent decay determined [3] that a fraction of excitons participated in slow $(\approx 10 \mu \mathrm{s})$ energy transfer. Moreover, such slow energy transfer from PNP to PtOEP was not found in the photoluminescent transient response of PtOEP:PNP. Thus, it was inferred that slow Dexter transfer of host triplet excitons was responsible for the difference between the photoluminescent and electroluminescent decay transients. By separating the electroluminescent decay into components exhibiting fast or slow energy transfer, it was determined [3] that in the $0.1 \%$ PtOEP:PNP films only 0.4 triplets are transferred to PtOEP for every singlet exciton. Since the expected [6] singlet-to-triplet ratio in the host material is $1: 3$, triplet energy transfer is evidently inefficient in this system. 
Owing to long lifetimes, triplet diffusion lengths in organic materials may also be substantially longer than for singlets. Indeed, it has been found [6] that in $\mathrm{Alq}_{3}$, the triplet diffusion length is $>1400 \AA$, as compared to singlet diffusion lengths of $\approx 100 \AA$. To optimize efficiency, phosphorescent OLEDs can be modified to trap triplets within the luminescent layer, thereby increasing the probability for energy transfer from the host to the phorphor. A material suitable for this purpose is 2,9-dimethyl-4,7 diphenyl1,10-phenanthroline (bathocuproine, or BCP), which has previously been used as a hole blocking layer in OLEDs [27]. When placed between a doped HTL and an Alq $_{3}$ ETL, it was found that light emission originated from the HTL. As confirmed by the proposed energy level diagram [28] of Fig. 5, BCP has a large ionization potential and blocks the passage of holes out of the HTL. These results also suggest that the lowest unoccupied molecular orbital (LUMO) level of BCP freely allows the transport of electrons resulting in exciton formation in the HTL. Furthermore, since the energy gap in BCP is $\approx 3.5 \mathrm{eV}$, it should act as a barrier to exciton diffusion.

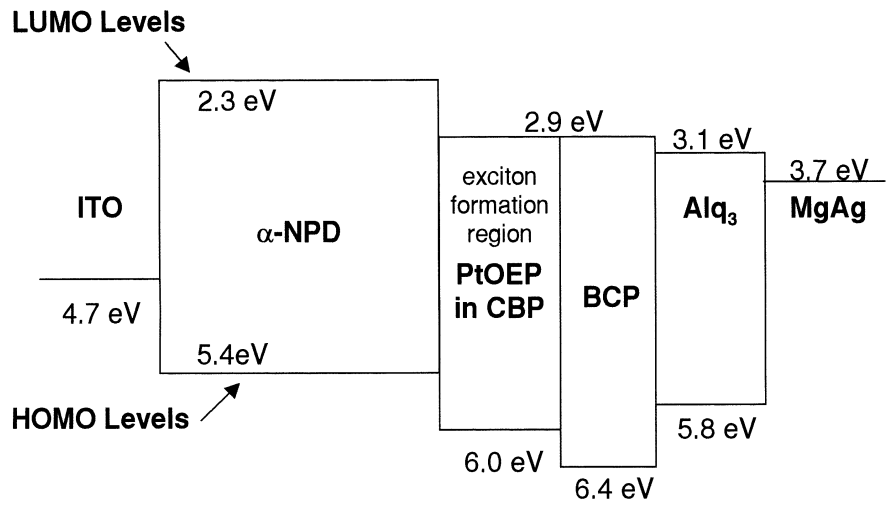

(a)

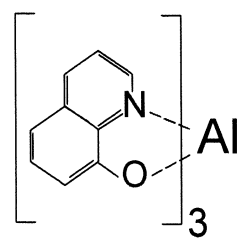

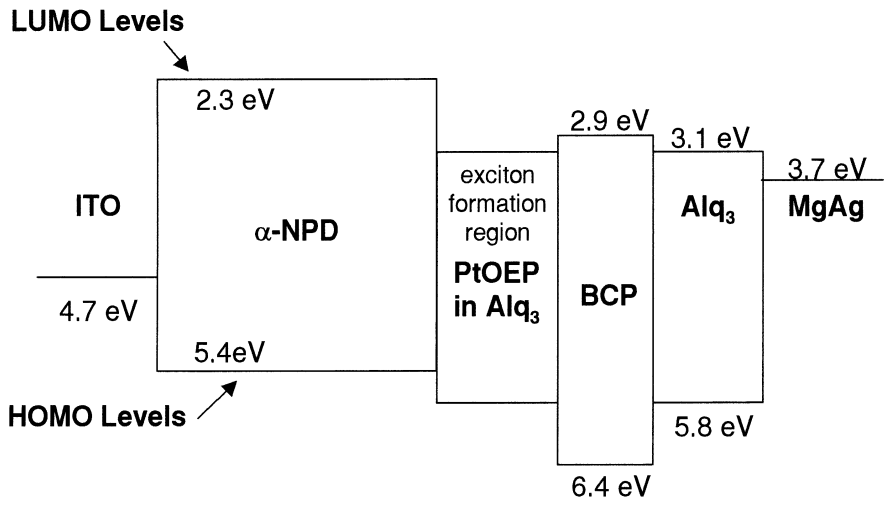

(b)<smiles>C1CCC(N2CCCC(N3CC4CCCCC4C4(CCCCC4)C3)C2)CC1</smiles>

(c)

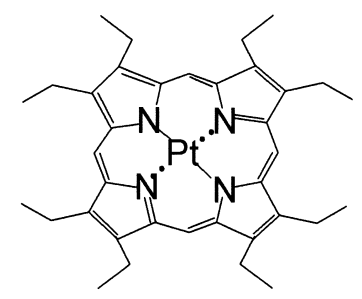

(d)

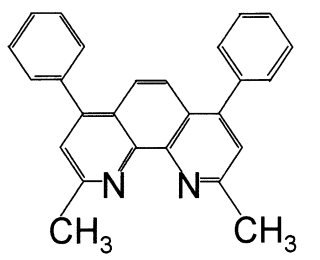

Fig. 5 Proposed energy level diagram of the electroluminescent devices containing PtOEP. The luminescent region is sandwiched between electron blocking $\alpha$-NPD and hole blocking BCP. Also shown are the chemical structures of (a) $\mathrm{Alq}_{3}$, (b) $\mathrm{CBP}$, (c) PtOEP and (d) BCP.

The electroluminescent efficiency of PtOEP can also be optimized by the selection of the host material. For example, the photoluminescent efficiency of PtOEP in a $4,4^{\prime}-\mathrm{N}, \mathrm{N}^{\prime}$-dicarbazole-biphenyl (CBP) host is approximately twice that of PtOEP in an $\mathrm{Alq}_{3}$ host [29]. The external quantum efficiencies of several OLEDs employing these hosts and a BCP barrier layer are shown in Fig. 6. As expected, the combination of a CBP host and a BCP barrier layer maximizes PtOEP emission. The benefits of the BCP barrier are most noticeable for the thinner (250 $)$ luminescent layers, where energy collection by PtOEP is particularly inefficient. Here, the quantum efficiency in CBP doped devices is $\eta=(2.2 \pm 0.1) \%$ at $100 \mathrm{~cd} / \mathrm{m}^{2}$, which is nearly twice the reported best result of $1.3 \%$ in PtOEP:Alq ${ }_{3}$ devices [1]. The peak efficiency of $\eta=(5.6 \pm 0.1) \%$ for the PtOEP:CBP device is equivalent to an internal quantum efficiency of $\approx 32 \%$ [30], higher than is expected if only singlet excitons are transferred to PtOEP molecules [6]. BCP is less useful for devices employing PtOEP in 
an $\mathrm{Alq}_{3}$ host where previous studies [1] have shown that under optimum conditions, energy transfer is nearly complete with up to $\approx 90 \%$ of excitons transferred to the dye within a luminescent layer with a thickness of $400 \AA$.

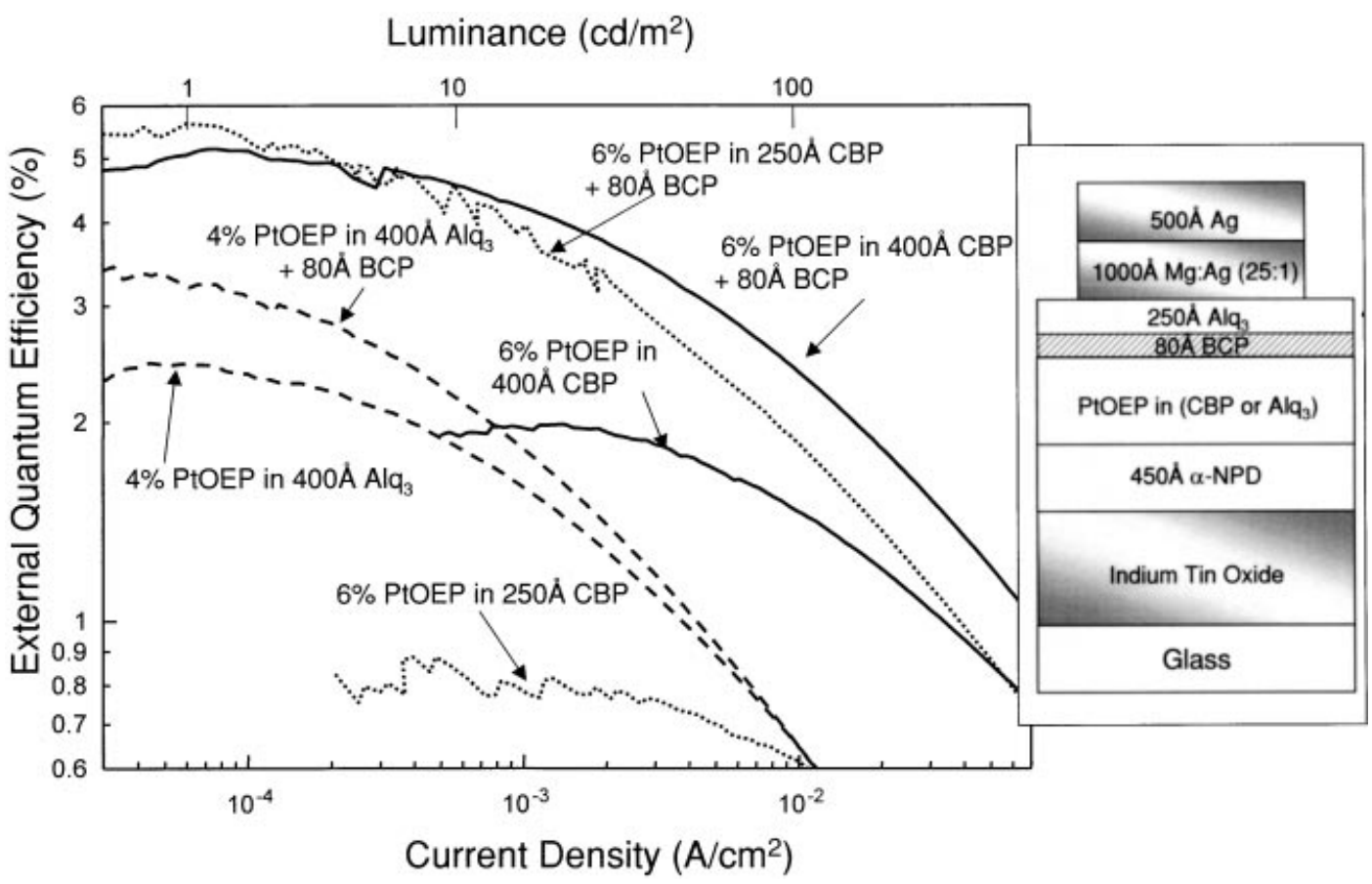

Fig. 6 External quantum efficiencies of PtOEP:CBP and PtOEP:Alq ${ }_{3}$ devices as a function of current with and without a BCP blocking layer. The top axis shows the luminance of the device with a $80 \AA$ thick BCP layer and a $400 \AA$ thick CBP luminescent layer doped with 6\% PtOEP. Inset: Schematic cross section of the high efficiency OLED, consisting of a $450 \AA$ thick 4,4'-bis[N-(1-napthyl)-N-phenyl-amino] biphenyl ( $\alpha$-NPD) HTL, PtOEP doped electron transport layer co-deposited with either $\mathrm{Alq}_{3}$ or CBP acting as the host, an $80 \AA$ thick layer of BCP and a further $200 \AA$ thick cap layer of $\mathrm{Alq}_{3}$ to prevent non-radiative quenching of PtOEP excitons at the cathode. Finally, a shadow mask with $1 \mathrm{~mm}$ diameter openings was used to define the cathode consisting of a $1000 \AA$ thick layer of 25:1 Mg:Ag, with a $500 \AA ̊$ thick Ag cap. From [29].

Unlike the case of PtOEP in $\mathrm{Alq}_{3}$, the triplet transfer efficiency of PtOEP in CBP is unknown. Indeed, the larger offset in HOMO and LUMO levels between PtOEP and CBP may encourage charge trapping and direct exciton formation on PtOEP molecules. Further work is required to clarify this issue. However, similar to PtOEP in $\mathrm{Alq}_{3}$, the maximum electroluminescent quantum efficiency for PtOEP in CBP is obtained at relatively high doping densities $(\approx 6-8 \%$ by mass $)$.

In Fig. 6, all the quantum efficiency curves are characterized by a rapid roll-off above a maximum of 'threshold' current density. At very high current densities, it is known that long phosphorescent lifetimes cause saturation of the emissive sites. This is particularly significant at low concentrations of the phosphorescent dopant, and is marked by an increase in emission from the host material. However, recent work [31] has demonstrated that the roll-off, which occurs at a much lower current density than saturation is due to triplet-triplet annihilation. For example, the efficiency of the $250 \AA$ thick CBP device begins to decrease at a lower current density than that of the $400 \AA$ thick CBP device (Fig. 6) due to the higher concentration of triplets within the thinner luminescent layer. The roll-off in efficiency also limits the maximum brightness obtainable in a phosphorescent OLED, and typically PtOEP-based devices are limited to $<1000 \mathrm{~cd} / \mathrm{m}^{2}$. This is a particular concern for passive matrix display applications, since these require OLEDs to be strongly excited by short electrical pulses. Unfortunately, triplet-triplet annihilation increases with the square of the triplet concentration [8]; thus unless the OLEDs can be pulsed faster than the phosphorescent decay rate, severe reductions in efficiency are likely. 
Triplet-triplet annihilation and saturation are minimized if the phosphorescent lifetime is short. This was demonstrated [32] using the green phosphorescent material fac tris(2-phenylpyridine) iridium $\left(\operatorname{Ir}(\mathrm{ppy})_{3}\right)[22,33-35]$. As in the case of PtOEP, $\operatorname{Ir}(\mathrm{ppy})_{3}$ was doped into a CBP host. The device structure and the proposed energy levels [28] of the charge transport materials are identical for $\operatorname{Ir}(\mathrm{ppy})_{3}: \mathrm{CBP}$ and PtOEP:CBP devices (see Fig. 5), although the ionization potentials of PtOEP and $\operatorname{Ir}(\mathrm{ppy})_{3}$ relative to their hosts are unknown. Once again, it was found that a thin $(60 \AA)$ BCP barrier layer was necessary to confine excitons within the luminescent zone and achieve high efficiencies.

Figure 7 shows the external quantum efficiencies of several $\operatorname{Ir}(\mathrm{ppy})_{3}$-based OLEDs. In contrast to PtOEP-based devices, the $\operatorname{Ir}(\text { ppy })_{3}$ doped devices exhibit a slow decrease in quantum efficiency with increasing current. In addition to the doped device, a heterostructure was fabricated where the luminescent region was a homogeneous film of $\operatorname{Ir}(\mathrm{ppy})_{3}$. The reduction in efficiency to $(\approx 0.8 \%)$ of neat $\operatorname{Ir}(\mathrm{ppy})_{3}$ is reflected in the transient decay, which has a lifetime of only $\approx 100 \mathrm{~ns}$ as compared with $\approx 500 \mathrm{~ns}$ in the $6 \% \operatorname{Ir}(\mathrm{ppy})_{3}$ in $\mathrm{CBP}$ devices.

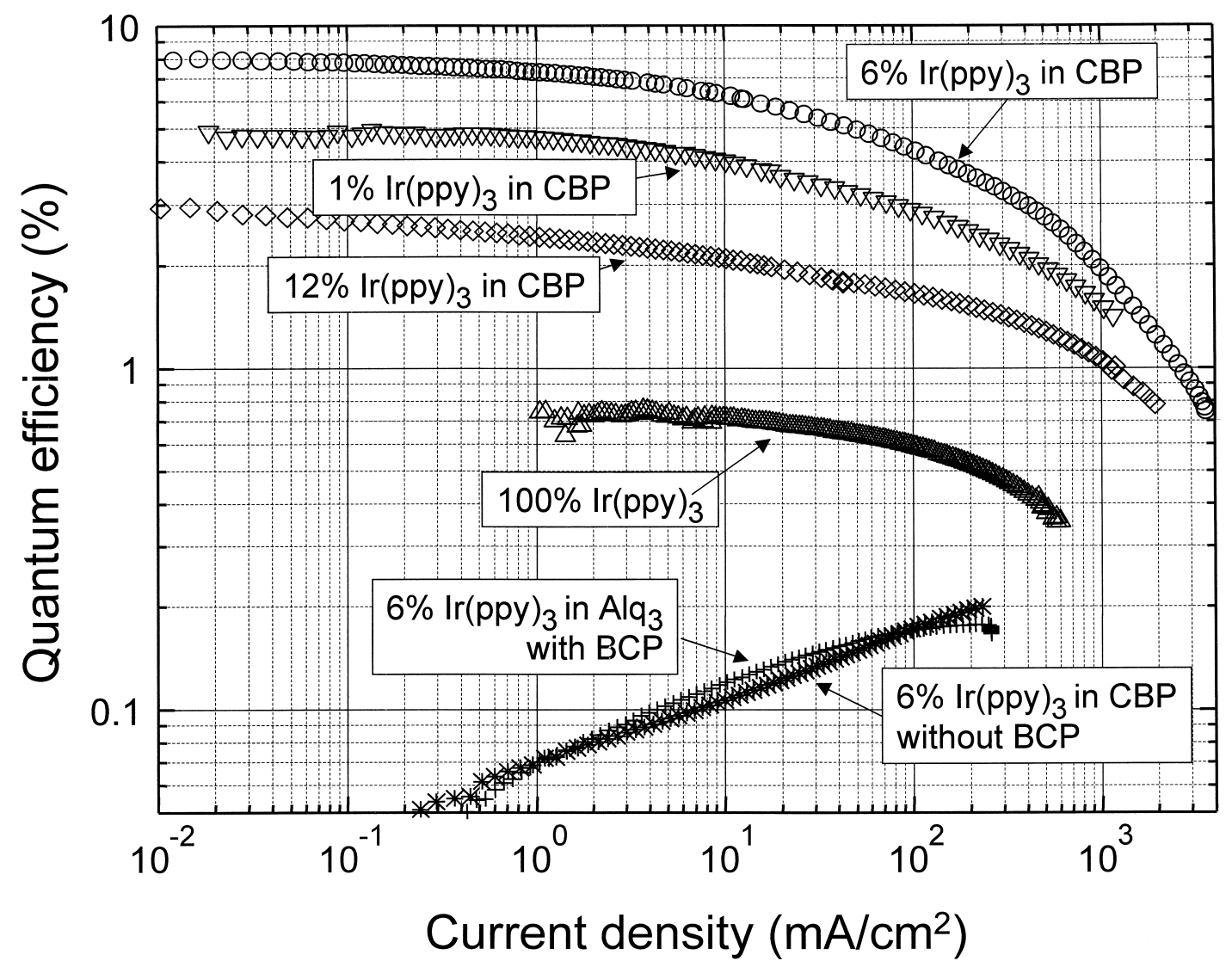

Fig. 7 The external quantum efficiency of OLEDs using $\operatorname{Ir}(\mathrm{ppy})_{3}: \mathrm{CBP}$ luminescent layers. Peak efficiencies are observed for mass ratio of $6 \% \operatorname{Ir}(\mathrm{ppy})_{3}: \mathrm{CBP}$. The $100 \% \operatorname{Ir}(\mathrm{ppy})_{3}$ device has a slightly different structure than shown in Fig. 1: the $\operatorname{Ir}(\mathrm{ppy})_{3}$ layer is $300 \AA$ thick and there is no BCP blocking layer. The efficiency of a $6 \%$ $\operatorname{Ir}(\mathrm{ppy})_{3}: \mathrm{CBP}$ device grown without a BCP layer is also shown. From [32].

In Fig. 8, the luminance and power efficiencies are plotted as functions of voltage. The peak power efficiency is $31 \mathrm{~lm} / \mathrm{W}$ with a quantum efficiency of $8 \%,(28 \mathrm{~cd} / \mathrm{A})$. At $100 \mathrm{~cd} / \mathrm{m}^{2}$, a power efficiency of $19 \mathrm{~lm} / \mathrm{W}$ with a quantum efficiency of $7.5 \%(26 \mathrm{~cd} / \mathrm{A})$ is obtained at a voltage of $4.3 \mathrm{~V}$. The transient response of $\operatorname{Ir}(\mathrm{ppy})_{3}$ in CBP is an approximately mono-exponential phosphorescent decay of $\approx 500 \mathrm{~ns}$, compared with a measured lifetime $[22,33,34]$ of $2 \mu$ s in degassed toluene at room temperature. Slow triplet relaxation can form a bottleneck in electrophosphorescence, thereby encouraging triplet-triplet annihilation [31] and saturation. But these lifetimes are short and result in only a gradual decrease in efficiency with increasing current, leading to a maximum luminance of $\approx 100000 \mathrm{~cd} / \mathrm{m}^{2}$. 


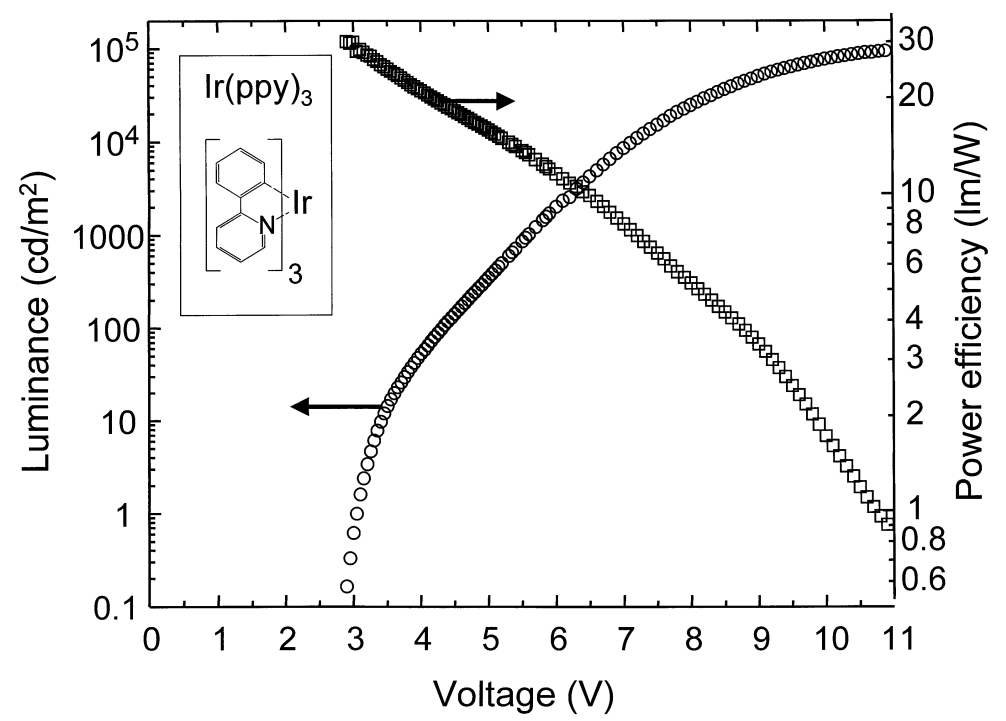

Fig. 8 The power efficiency and luminance of the $6 \% \operatorname{Ir}(\mathrm{ppy})_{3}: \mathrm{CBP}$ device. At $100 \mathrm{~cd} / \mathrm{m}^{2}$, the device requires $4.3 \mathrm{~V}$ and its power efficiency is $19 \mathrm{~lm} / \mathrm{W}$. Inset: The chemical structure of $\operatorname{Ir}(\mathrm{ppy})_{3}$. From [32].

In Fig. 9, the emission spectrum and CIE coordinates of $\operatorname{Ir}(\mathrm{ppy})_{3}$ are shown for the highest efficiency device. The peak wavelength is $\lambda=510 \mathrm{~nm}$, and the full width at half maximum is $70 \mathrm{~nm}$. The spectrum and CIE coordinates $(x=0.27, y=0.63)$ are independent of current. Even at very high current densities $\left(\approx 100 \mathrm{~mA} / \mathrm{cm}^{2}\right)$, blue emission from CBP is negligible - an indication of complete singlet energy transfer. As is the case of PtOEP in CBP, the triplet transfer efficiency is unknown and trapping and direct exciton formation on $\operatorname{Ir}(\mathrm{ppy})_{3}$ molecules cannot be ruled out.

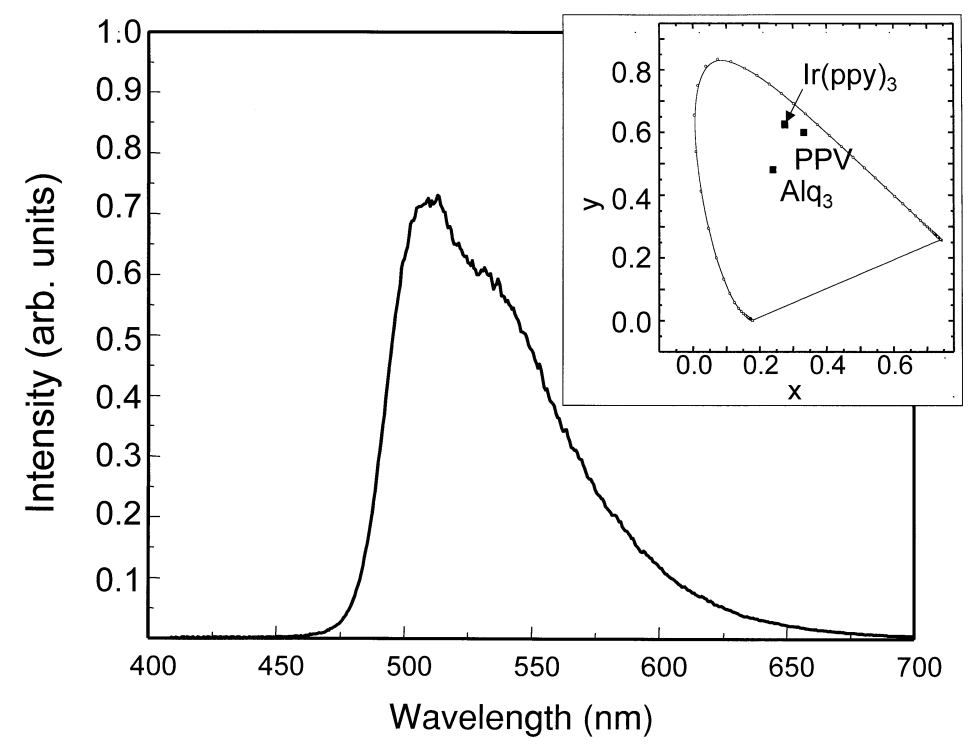

Fig. 9 The electroluminescent spectrum of $6 \% \operatorname{Ir}(\mathrm{ppy})_{3}: \mathrm{CBP}$. Inset: The Commission Internationale de L'Eclairage (CIE) chromaticity co-ordinates of $\operatorname{Ir}(\mathrm{ppy})_{3}$ in $\mathrm{CBP}$ are shown relative to the fluorescent green emitters $\mathrm{Alq}_{3}$ and poly(p-phenylenevinylene) (PPV). From [32].

We note that the device structure has the potential for further optimization. For example, the use of $\mathrm{LiF}$ cathodes [36,37], shaped substrates [30], and novel hole transport materials [38] that result in a reduction in operating voltage or increased quantum efficiency are also applicable to this work. These methods have yielded power efficiencies of $\approx 20 \mathrm{~lm} / \mathrm{W}$ in fluorescent small molecule devices [38]. The quantum 
efficiencies in these devices [39] at $100 \mathrm{~cd} / \mathrm{m}^{2}$ are typically $>5 \%$, and hence green-emitting electrophosphorescent devices with power efficiencies of $>40 \mathrm{~lm} / \mathrm{W}$ are anticipated.

\section{RELIABILITY OF PHOSPHORESCENT OLEDS}

The suitability of semiconducting organic thin films for practical use is ultimately determined by device reliability. Unless OLEDs can demonstrate thousands of hours of high performance then applications will be scarce. But fluorescent OLEDs have already achieved such standards [40] and there is no reason to believe that phosphorescent materials should be any less stable; they may in fact improve reliability. For example, there has been speculation that molecules in the triplet state may be particularly susceptible to degradation because of the long lifetime of the excitation [41]. Although the triplet lifetime of phosphors possessing significant ISC may approach $\approx 100 \mu \mathrm{s}$, these lifetimes are still much shorter than those in fluorescent materials, where the triplet decay is strongly forbidden. Thus, it is possible that by acting as sinks for triplet excitations, phosphorescent materials may in fact ultimately improve device reliability.

Owing to the novelty of phosphorescent dyes, there is little data to test such a contention. But preliminary reliability data is available for PtOEP-based OLEDs, and a lifetime of $>10^{5} \mathrm{~h}$ at a luminance of $35 \mathrm{~cd} / \mathrm{m}^{2}$ is observed for OLEDs incorporating PtOEP. This is at least as reliable as the longest lived fluorescent devices employing all the same materials except PtOEP. Another study [42] has identified a derivative of PtOEP for oxygen sensing applications, where long operational lifetimes are required in relatively uncontrolled environments.

\section{CONCLUSION}

Although this work has highlighted the performance advantages inherent to phosphorescence, these advantages are eliminated if the phosphor cannot efficiently gather triplet and singlet excitons within the device. For example, the lanthanide complexes exhibit losses in energy transfer within the complex, and also between the host and the complex; and unless these deficiencies can be overcome it is unlikely that lanthanide complexes will find application in OLEDs. In contrast, efficient triplet energy transfer and possibly direct charge trapping and exciton formation is partly responsible for the success of organometallic compounds such as PtOEP and $\operatorname{Ir}(\mathrm{ppy})_{3}$. Since organometallic complexes featuring L-S coupling are the most successful phosphors to date, similar complexes that phosphoresce at different wavelengths deserve investigation. In particular, blue phosphorescent OLEDs present a challenge since wide-gap $(\approx 3.5 \mathrm{eV})$ host materials and luminescent complexes will be required to overcome exchange energy losses. It is clear, however, that the efficiency improvements offered by phosphorescence outweigh the slight increase in voltage that results from the use of large-energy-gap materials. Furthermore, triplet energy transfer remains poorly characterized, and if these mechanisms can be better understood improvements in device structure and host material combinations may yet result in further enhancements in phosphorescent device performance.

\section{ACKNOWLEDGEMENT}

We thank Dr C. Adachi for many useful discussions. This work was funded by Universal Display Corporation, DARPA, AFOSR and NSF.

\section{REFERENCES}

1 M. A. Baldo, D. F. O’Brien, Y. You, A. Shoustikov, S. Sibley, M. E. Thompson, S. R. Forrest. Nature 395, 151 (1998).

2 Y. Ma, H. Zhang, J. Shen, C. Che. Synth. Met. 94, 245 (1998).

3 V. Cleave, G. Yahioglu, P. Le Barny, R. Friend, N. Tessler. Adv. Mat. 11, 285 (1999).

4 S. Hoshino, H. Suzuki. Appl. Phys. Lett. 69, 224 (1996).

5 J. Kido, K. Nagai, Y. Ohashi. Chem. Lett. 657 (1990).

6 M. A. Baldo, D. F. O'Brien, M. E. Thompson, S. R. Forrest. Phys. Rev. B 60, 14422 (1999).

7 C. W. Tang, S. A. VanSlyke, C. H. Chen. J. Appl. Phys. 65, 3610 (1989). 
8 M. Klessinger, J. Michl. Excited States and Photochemistry of Organic Molecules. VCH Publishers, New York (1995).

9 T. Forster. Disc. Faraday Soc. 27, 7 (1959).

10 V. L. Ermolaev, E. B. Sveshnikova. Doklady Akademii Nauk SSSR 148, 1295 (1963).

11 M. A. Baldo, M. E. Thompson, S. R. Forrest. Nature 403, 750 (2000).

12 D. L. Dexter. J. Chem. Phys. 21, 836 (1953).

13 S. P. Sinha. Complexes of the Rare Earths. Pergamon, Oxford (1966).

14 J. Kido, K. Nagai, Y. Okamoto, T. Skotheim. Chem. Lett. 1267 (1991).

15 J. Kido, H. Hayese, K. Hongawa, K. Nagai, K. Okuyama. Appl. Phys. Lett. 65, 2124 (1994).

16 R. J. Curry, W. P. Gillin. Appl. Phys. Lett. 75, 1380 (1999).

17 C. Adachi, T. Tsutsui, S. Saito. Appl. Phys. Lett. 55, 1489 (1989).

18 M. L. Bhaumik, M. A. El-Sayed. J. Chem. Phys. 42, 787 (1965).

19 F. J. Steemers, W. Verboom, D. N. Reinhoudt, E. B. van der Tol, W. Verhoeven. J. Am. Chem. Soc. 117, 9408 (1995).

20 P. W. Atkins, R. S. Friedman. Molecular Quantum Mechanics. Oxford University Press, New York (1997).

21 D. B. Papkovski. Sens. and Actuators B 29, 213 (1995).

22 E. Vander Donckt, B. Camerman, F. Hendrick, R. Herne, R. Vandeloise. Bull. Soc. Chim. Belg. 103, 207 (1994).

23 Y. Ma, C.-M. Che, H.-Y. Chao, X. Zhou, W.-H. Chan, J. Shen. Adv. Mat. 11, 852 (1999).

24 A. Mills, A. Lepre. Anal. Chem. 69, 4653 (1997).

25 G. Ponterini, N. Serpone, M. A. Bergkamp, T. L. Netzel. J. Am. Chem. Soc. 105, 4639 (1983).

26 V. Bulovic, A. Shoustikov, M. A. Baldo, E. Bose, V. G. Kozlov, M. E. Thompson, S. R. Forrest. Chem. Phys. Lett. 287, 455 (1998).

27 Y. Kijima. In Mat. Res. Soc., San Francisco, California (1998).

28 I. G. Hill, A. Kahn. J. Appl. Phys. 86, 4515 (1999).

29 D. F. O’Brien, M. A. Baldo, M. E. Thompson, S. R. Forrest. Appl. Phys. Lett. 74, 442 (1999).

30 G. Gu, D. Z. Garbuzov, P. E. Burrows, S. Venkatesh, S. R. Forrest, M. E. Thompson. Op. Lett. 22, 396 (1997).

31 M. A. Baldo, C. Adachi, S. R. Forrest. (1999). Unpublished.

32 M. A. Baldo, S. Lamansky, P. E. Burrows, M. E. Thompson, S. R. Forrest. Appl. Phys. Lett. 75, 4 (1999).

33 K. A. King, P. J. Spellane, R. J. Watts. J. Am. Chem. Soc. 107, 1431 (1985).

34 K. Dedeian, P. I. Djurovich, F. O. Garces, G. Carlson, R. J. Watts. Inorg. Chem. 30, 1685 (1991).

35 M. G. Colombo, T. C. Brunold, T. Riedener, H. U. Gudel, M. Fortsch, H.-B. Burgi. Inorg. Chem. 33, 545 (1994).

36 L. S. Hung, C. W. Tang, M. G. Mason. Appl. Phys. Lett. 70, 152 (1997).

37 G. E. Jabbour, Y. Kawabe, S. E. Shaheen, J. F. Wang, M. M. Morrell, B. Kippelen, N. Peyghambarian. Appl. Phys. Lett. 71, 1762 (1997).

38 B. Kippelen, G. E. Jabbour, S. E. Shaheen, et al. In Mat. Res. Soc., San Francisco, California (1999).

39 J. Kido, Y. Iizumi. Appl. Phys. Lett. 73, 2721 (1998).

40 J. Shi, C. W. Tang. Appl. Phys. Lett. 70, 1665 (1997).

41 G. Sakamoto, C. Adachi, T. Koyama, Y. Taniguchi, C. D. Merrit, H. Murata, Z. H. Kafafi. Appl. Phys. Lett. 75 , 766 (1999).

42 S.-K. Lee, I. Okura. Anal. Comm. 34, 185 (1997). 